\title{
Hydrodynamic simulation of supernova remnants including efficient particle acceleration
}

\author{
D. C. Ellison ${ }^{1}$, A. Decourchelle ${ }^{2}$, and J. Ballet ${ }^{2}$ \\ 1 Department of Physics, North Carolina State University, Box 8202, Raleigh NC 27695, USA \\ 2 Service d'Astrophysique, DSM/DAPNIA, CEA Saclay, 91191 Gif-sur-Yvette, France \\ e-mail: adecourchelle@cea.fr, jballet@cea.fr
}

Received 11 July 2003 / Accepted 14 August 2003

\begin{abstract}
A number of supernova remnants (SNRs) show nonthermal X-rays assumed to be synchrotron emission from shock accelerated $\mathrm{TeV}$ electrons. The existence of these $\mathrm{TeV}$ electrons strongly suggests that the shocks in SNRs are sources of galactic cosmic rays (CRs). In addition, there is convincing evidence from broad-band studies of individual SNRs and elsewhere that the particle acceleration process in SNRs can be efficient and nonlinear. If SNR shocks are efficient particle accelerators, the production of CRs impacts the thermal properties of the shock heated, X-ray emitting gas and the SNR evolution. We report on a technique that couples nonlinear diffusive shock acceleration, including the backreaction of the accelerated particles on the structure of the forward and reverse shocks, with a hydrodynamic simulation of SNR evolution. Compared to models which ignore CRs, the most important hydrodynamical effects of placing a significant fraction of shock energy into CRs are larger shock compression ratios and lower temperatures in the shocked gas. We compare our results, which use an approximate description of the acceleration process, with a more complete model where the full $\mathrm{CR}$ transport equations are solved (i.e., Berezhko et al. 2002), and find excellent agreement for the CR spectrum summed over the SNR lifetime and the evolving shock compression ratio. The importance of the coupling between particle acceleration and SNR dynamics for the interpretation of broad-band continuum and thermal X-ray observations is discussed.
\end{abstract}

Key words. ISM: cosmic rays - acceleration of particles - shock waves - ISM: supernova remnants

\section{Introduction}

It is commonly believed that the shocks in supernova remnants (SNRs) produce the majority of galactic cosmic rays (CRs) with energies below $\sim 10^{15} \mathrm{eV}$ via diffusive shock acceleration (e.g., Drury 1983; Blandford \& Eichler 1987). Convincing support for the production of $\mathrm{TeV}$ electrons in SNRs comes from the synchrotron interpretation of nonthermal X-ray emission (e.g., Reynolds 1998) observed in an increasing number of young SNRs such as SN1006 (Koyama et al. 1995), Cas A (Allen et al. 1997), G347.3-0.5 (Slane et al. 1999), and RCW 86 (Borkowski et al. 2001) ${ }^{1}$.

In addition to their putative role in accelerating cosmic rays, the shocks in SNRs heat the ambient interstellar medium and ejecta to X-ray emitting temperatures. The interpretation of these X-ray observations leads to inferences for important

Send offprint requests to: D. Ellison,

e-mail: don_ellison@ncsu.edu

1 As of this writing, there is no unambiguous evidence for the production of TeV ions in SNRs (see Berezhko et al. 2002,for a discussion of SN1006 in this regard). The recent claim that TeV emission from SNR RX J1713.7-3946 (also called G347.3-0.5) observed by CANGAROO II is from pion-decay (e.g., Enomoto et al. 2002) is still under debate (see, for example, Reimer \& Pohl 2002; Butt et al. 2002). quantities such as the supernova (SN) explosion energy, ejecta mass and composition, ambient densities, shock speed, and rate of electron and proton equilibration. That CR production and thermal heating in SNRs may be coupled comes from the fact that diffusive shock acceleration is intrinsically efficient in high Mach number shocks if even a small fraction of the shock heated plasma is injected into the acceleration process (e.g., Ellison \& Eichler 1984; Jones \& Ellison 1991; Berezhko \& Ellison 1999; Malkov 1998; Blasi 2002).

If strong coupling between particle acceleration and shock heating occurs, the modeling of efficient particle acceleration in SNRs offers the possibility of using the high-quality X-ray and $\gamma$-ray data currently being collected by spacecraft (e.g., Chandra, XMM-Newton, INTEGRAL) to address fundamental questions concerning the SNR origin of CRs and the underlying physics of diffusive shock acceleration, particularly the injection of thermal particles into the acceleration process. Furthermore, strong coupling implies that the inferences made from X-ray observations may differ substantially between interpretations which include particle acceleration selfconsistently and those that do not.

Despite the expected efficiency of diffusive shock acceleration, X-ray spectra from SNRs have generally been modeled 
and interpreted assuming that the shocks place an insignificant fraction of their energy in cosmic rays. Exceptions to this include the early works of Chevalier (1983), Heavens (1984), and Boulares \& Cox (1988). Chevalier (1983) investigated the effects of cosmic-ray pressure on SNR dynamics using a two-fluid, self-similar solution with an arbitrary fraction of thermal gas (adiabatic index $\gamma=5 / 3$ ) and relativistic gas $(\gamma=4 / 3)$. More recent work has been done by Dorfi $\&$ Böhringer (1993) and Dorfi (1994). In our preliminary work (i.e., Decourchelle et al. 2000), we developed a model which coupled the approximate nonlinear (NL) acceleration calculation of Berezhko \& Ellison (1999) with an analytic, self-similar description of the SNR hydrodynamics (i.e., Chevalier 1983) and a non-equilibrium ionization calculation of X-ray emission (e.g., Decourchelle \& Ballet 1994). We illustrated this model by fitting ASCA and RXTE observations of Kepler's SNR and found adequate fits when acceleration was efficient at the forward shock but inefficient at the reverse shock.

The effects of efficient particle acceleration on SNR hydrodynamics where calculated in a hydro computer simulation of SNRs by Blondin \& Ellison (2001). This was done by globally changing the effective ratio of specific heats, $\gamma_{\mathrm{eff}}$, from $5 / 3$ to values approaching 1 , but did not include coupling between the acceleration and the hydro. As $\gamma_{\text {eff }}$ was decreased, the shocked gas became more compressible, the shock compression ratio increased, and the interaction region between the forward and reverse shocks narrowed. Blondin \& Ellison (2001) were able to show in two and three-dimensional simulations that if the interaction region was narrow enough, convective instabilities produced Rayleigh-Taylor fingers of dense ejecta material which were able to reach and perturb the forward shock.

Here, we introduce and describe in detail a CR-Hydro model which uses the same NL acceleration calculation of Berezhko \& Ellison (1999), but replaces the self-similar description used in Decourchelle et al. (2000) with a 1D hydro simulation such as that used by Blondin \& Ellison (2001). The simulation is more general than the analytic approach used by Decourchelle et al. (2000) since it is not restricted to selfsimilar evolution and allows for a continuous change in the acceleration efficiency as the SNR evolves. We show, however, that when acceleration efficiency is nearly constant during the self-similar phase, the two models closely correspond, providing an important check on the validity of both models. While we do not calculate X-ray thermal spectra in this paper, we show, with various examples, how the efficient production of CR protons influences SNR evolution and discuss the implications this has on the interpretation of X-ray observations.

\section{CR-hydro model}

Our CR-hydro model couples a spherically symmetric hydrodynamic simulation with a calculation of nonlinear diffusive shock acceleration. The particle acceleration calculation determines how energy is divided between the thermal gas and relativistic particles, and provides the particle distribution over all energies behind the shock, as well as the effective ratio of specific heats, $\gamma_{\mathrm{eff}}$, defined below. In future work, we will use the electron and ion distribution functions to calculate the broad-band continuum photon emission from radio to $\mathrm{TeV}$ energies (e.g., Ellison et al. 2001), and use the self-consistent thermal properties in a non-equilibrium calculation of X-ray lines (e.g., Decourchelle et al. 2000). For now, we restrict ourselves to calculating $C R$ proton spectra integrated over the SNR lifetime.

\subsection{Hydrodynamic simulation}

We use a standard hydrodynamic simulation in one dimension to model the effects of a supernova explosion in the interstellar medium (ISM) (see Blondin \& Ellison 2001, and references therein). We are free to choose arbitrary ejecta and ISM mass density profiles, but to facilitate our comparisons discussed below, we adopt the parameters determined for SN1006 by Berezhko et al. (2002), i.e., we assume a constant density, constant temperature ISM, and take the initial ejecta density profile to be $\rho \propto r^{-n}$, with $n=7$ and a constant density plateau at small radii (i.e., Chevalier 1982) ${ }^{2}$. While the modeling of particular, young SNRs depends critically on the ejecta and ISM densities (e.g., Decourchelle et al. 2000), the general character of the results we present here are insensitive to these details. Specifically, we begin at some time $t_{\text {start }}<0.1 t_{\text {ch }}$ with undisturbed ejecta and ISM separated by a contact discontinuity. Here, $t_{\mathrm{ch}}=R_{\mathrm{ch}} / V_{\mathrm{ch}}$ is the characteristic age with $R_{\mathrm{ch}}=$ $\left[3 M_{\mathrm{ej}} /\left(4 \pi \rho_{0}\right)\right]^{1 / 3}, V_{\mathrm{ch}}=\sqrt{2 E_{\mathrm{sn}} / M_{\mathrm{ej}}}$, and $\rho_{0}=\left(1+4 f_{\mathrm{He}}\right) m_{\mathrm{p}} n_{\mathrm{p} 0}$, where $E_{\mathrm{sn}}$ is the explosion energy, $M_{\mathrm{ej}}$ is the ejecta mass, $n_{\mathrm{p} 0}$ is the ISM proton number density, $f_{\mathrm{He}}$ is the helium to proton number ratio, and $m_{\mathrm{p}}$ is the proton mass ${ }^{3}$.

The magnetic field, $B$, is ignored in our hydrodynamic model (we implicitly assume that $B^{2} / 8 \pi$ is small compared to the thermal pressure), but it is an important parameter for the particle acceleration discussed next.

\subsection{Nonlinear diffusive shock acceleration}

The full details of the nonlinear acceleration model used here are given in Berezhko \& Ellison (1999) and Ellison et al. (2000). This is an approximate, algebraic model of diffusive shock acceleration containing the essential physics of NL acceleration, but which parameterizes important properties of the process such as the injection efficiency and the maximum energy particles achieve. While more complete models of nonlinear shock acceleration exist (e.g., Jones \& Ellison 1991; Berezhko et al. 1996; Malkov \& Drury 2001), our algebraic approximation is easier to include in global models of SNRs. It is computationally fast making it far less time consuming to do parameter searches and to compare model results with observations. In Sect. 2.5 we show by direct comparison that it gives similar results to the more physically complete model of Berezhko et al. (2002).

Briefly, the nonlinear effects in diffusive shock acceleration are: (i) the self-generation of magnetic turbulence by counterstreaming energetic particles. Back streaming particles produce

\footnotetext{
${ }^{2}$ At the start of the simulation, the ejecta temperature is assumed to be low enough to be insignificant.

3 Throughout this paper the subscript 0 (2) implies values upstream (downstream) from the shock.
} 
turbulence in the magnetic field which leads to stronger scattering of the particles and hence to more acceleration, quickly leading to saturated turbulence levels near $\delta B / B \sim 1$ in strong shocks; (ii) the modification (i.e., smoothing) of the shock precursor by the backpressure of energetic particles. The precursor influences the subshock compression, $r_{\text {sub }}$, the injection and acceleration efficiencies, and the shape of the accelerated spectrum. Since particle diffusion lengths are generally increasing functions of momentum (e.g., Blandford \& Eichler 1987; Giacalone et al. 1993), high momentum particles sample a broader portion of the flow velocity profile, and hence experience larger effective total compression ratios, $r_{\text {tot }}$, than low momentum particles. Consequently, higher momentum particles have a flatter power-law index than those at lower momenta and can dominate the pressure in a NL fashion. The resultant superthermal distribution has a characteristic concave upward curvature until the spectrum turns over at the highest energies from losses (e.g., Ellison \& Eichler 1984; Blasi 2002; Malkov et al. 2002); and (iii) the increase in $r_{\text {tot }}$ from relativistic particle pressure and particle escape. As relativistic particles are produced and contribute significantly to the total pressure, their softer equation of state makes the shocked plasma more compressible $(\gamma \rightarrow 4 / 3)$. Even more important, as the highest energy particles escape from strong shocks they drain away energy flux which must be compensated for by ramping up the overall compression ratio to conserve the fluxes. Just as in radiative shocks, this is equivalent to $\gamma \rightarrow 1$ and $r_{\text {tot }}$ can become arbitrarily large (e.g., Kazanas \& Ellison 1986; Berezhko \& Ellison 1999; Malkov 1997). As the overall compression increases $\left(r_{\text {tot }}>4\right)$, the subshock compression ratio, $r_{\text {sub }}$, which is responsible for heating the gas, must become less than the test-particle (TP) value $\left(r_{\text {sub }}<4\right)$, causing the temperature of the shocked gas to drop below TP values. These changes in shock compression occur simultaneously with changes in the shape of the accelerated particle spectrum, thus linking $\mathrm{X}$-ray heating to cosmic-ray production. For reviews on diffusive shock acceleration see Drury (1983), Blandford \& Eichler (1987), Berezhko \& Krymsky (1988), Jones \& Ellison (1991), and Malkov \& Drury (2001).

The most important parameters associated with nonlinear shock acceleration are the Mach numbers (i.e., the shock speed, $u_{0}$, pre-shock hydrogen number density, $n_{\mathrm{p} 0}$, and preshock magnetic field, $B_{0}$ ), the injection efficiency, $\eta_{\text {inj }}$ (i.e., the fraction of total protons which end up with superthermal energies), and the maximum proton energy produced, $E_{\max }$. As described in Berezhko \& Ellison (1999), our model includes Alfvén heating in the precursor which reduces the efficiency compared to adiabatic heating and makes the magnetic field strength an important parameter. For given sonic and Alfvén Mach numbers (i.e., given $M_{\mathrm{S}}=\sqrt{\rho_{0} u_{0}^{2} /\left(\gamma P_{0}\right)}$ and $\left.M_{\mathrm{A}}=\sqrt{4 \pi \rho_{0} u_{0}^{2}} / B_{0}\right)$, and a given shock size and age, $\eta_{\text {inj }}$ sets the overall acceleration efficiency and determines the importance of NL effects. With other parameters fixed, Alfvén wave heating causes the acceleration efficiency to decrease with increasing $B_{0}$. In a complete model of diffusive shock acceleration, the injection efficiency would be determined from first principles. However, no current model of diffusive shock acceleration can do this and injection remains dependent on approximations of poorly understood wave-particle interactions ${ }^{4}$. Here, we investigate the effects of efficient acceleration by varying $\eta_{\text {inj. }}$. A principle aim of future work is to constrain $\eta_{\text {inj }}$ from models using X-ray and broad-band observations of particular SNRs.

In order to compare our results directly to those of Berezhko et al. (2002), we assume as they do that the magnetic field is turbulent, adopting the Bohm limit for strong particle scattering, and somewhat arbitrarily take the field downstream from the shock to be the compressed upstream magnetic field i.e., $B_{2}=r_{\text {tot }} B_{0}$, where $B_{0}\left(B_{2}\right)$ is the upstream (downstream) magnetic field strength. We do not expressly consider shock obliquity, i.e., the angle between the local shock normal and $B_{0}$, even though this may be an important factor for understanding emission around the rims of some SNRs (see Reynolds 1998, for a discussion of the effects of shock obliquity in a test-particle description of particle acceleration in SNRs). As a crude approximation, we could model the asymmetry seen in many SNRs, including SN 1006, by combining results for different quadrants of the remnant where values of the magnetic field and injection parameter were varied.

In our examples presented here, we take the unshocked ISM field to be $B_{0}=20 \mu \mathrm{G}$ to match the value determined by Berezhko et al. (2002) for SN 1006. For simplicity, unless explicitly stated we use the same constant value for the field in the unshocked supernova ejecta even though, in reality, this field is likely to weaken considerably with time because of flux conservation (in the discussions associated with Figs. 4 (Sect. 2.3) and 8 (Sect. 2.4), we show some effects of a weak ejecta field).

The maximum energy cosmic rays obtain depends, in part, on the scattering mean free path, $\lambda$, which is assumed to be,

$\lambda=\eta_{\mathrm{mfp}} r_{\mathrm{g}}$

where $\eta_{\mathrm{mfp}} \gtrsim 1$ is taken to be a constant and $r_{\mathrm{g}}=p /(q B)$ is the gyroradius in SI units. Small values of $\eta_{\mathrm{mfp}}$ imply strong scattering and allow higher maximum proton energies in a given system. The Bohm limit implies $\eta_{\mathrm{mfp}} \simeq 1$.

The phase-space momentum distributions for protons, $f(p)$, are calculated as in Ellison et al. (2000) and consist of a

4 While in principle plasma simulations, where particles move in response to Newton's and Maxwell's equations (particle-in-cell), can fully describe wave-particle interactions and injection, in practice, they have not yet done so because of computational limits. The limits have been insurmountable for two main reasons: (i) The simulations must be performed fully in $3 \mathrm{D}$ because 1 - or $2 \mathrm{D}$ simulations unphysically prevent cross-field diffusion (Jokipii et al. 1993; Jones et al. 1998). In all cases except strictly parallel shocks (where the upstream magnetic field is parallel to the shock normal), cross-field diffusion will be an essential part of the injection and acceleration process; and (ii) in order for NL effects to become apparent or field amplification to occur on large scales, the simulations must be run long enough in a large enough box with enough particles for a significant population of superthermal particles to be produced. If, in addition, electrons are to be investigated, the simulations must use the short electron timestep yet run for many proton time-scales increasing computation time considerably. 


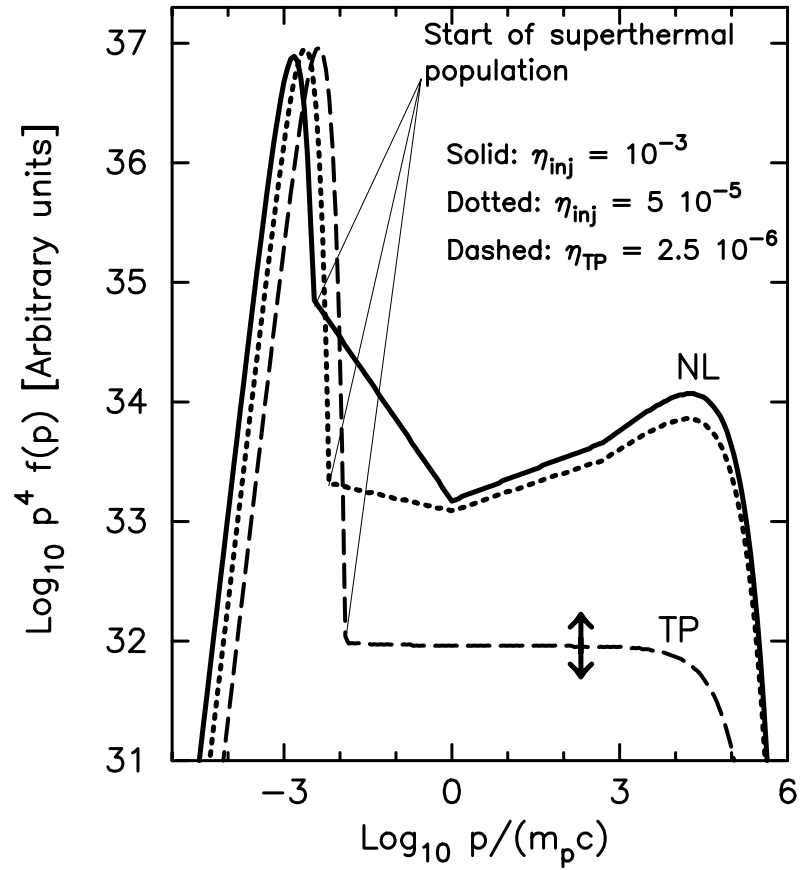

Fig. 1. Schematic particle distribution functions, $f(p)$, versus momentum, $p$, where $p^{4} f(p)$ is plotted to emphasize the spectral curvature in the nonlinear results (solid and dotted curves). The kinks in these spectra are manifestations of the piecewise approximation used in Berezhko \& Ellison (1999). The lowest momentum kink separates the thermal from superthermal population. Above that is the threecomponent power law, and the turnover at the highest momentum. The up and down arrow on the test-particle result (dashed curve) indicates that there is no constraint in TP models on the density of superthermal particles other than that their energy density be insignificant.

thermal component, a three-component power law at superthermal energies, and a turnover at the highest energies given by

$\exp \left[-\frac{1}{\alpha}\left(\frac{p}{p_{\max }}\right)^{\alpha}\right]$

Here, $\alpha$ is a constant and $p_{\max }=E_{\max } / c$ is determined by setting the acceleration time equal to the SNR age, $t_{\mathrm{snr}}$, or by setting the diffusion length of the highest energy particles equal to some fraction, $f_{\mathrm{sk}}$, of the shock radius, $R_{\mathrm{sk}}$, whichever gives the lowest $p_{\max }$ (see Baring et al. 1999). With these assumptions, $p_{\max }$ is proportional to the magnetic field strength. Reynolds (1998) has shown that when synchrotron emission is summed over space in a spherically symmetric SNR model, the resultant photon spectrum tends to be broader than that produced by a single electron distribution falling off as $\exp \left(-p / p_{\max }\right)$. This behavior can be approximated in our model by varying $\alpha$ and $\alpha \sim 0.5$ has been used to fit X-ray and radio spectra in particular SNRs (e.g., Berezhko et al. 1999; Ellison et al. 2001). However, Berezhko et al. (2002) obtain a good fit to SN1006 with a sharp turnover in $f(p)$ and to match their results, we take $\alpha=4$.

Figure 1 illustrates the essential differences in particle spectra between TP shock acceleration (dashed line) and NL acceleration with different injection efficiencies (solid and dotted lines). Compared to the TP power law, i.e.,

$f(p) \mathrm{d}^{3} p \propto p^{-\sigma} \mathrm{d}^{3} p \quad$ with $\quad \sigma=\frac{3 r_{\text {tot }}}{r_{\text {tot }}-1}$,

the high-energy portion $\left(p>m_{\mathrm{p}} c\right)$ of a NL spectrum is flatter, the low-energy portion of the superthermal spectrum (above the thermal peak and below $m_{\mathrm{p}} c$ ) is steeper, and the thermal part is at a lower temperature. In the TP approximation, the normalization of the power-law portion of the spectrum (relative to the thermal peak) is arbitrary as long as it is low enough to contain an insignificant fraction of the total energy. If we arbitrarily set this fraction at 0.01 , we can determine, for a given set of shock parameters, the injection efficiency, $\eta_{\mathrm{TP}}$, below which the thermal gas is decoupled from the power law, i.e., for any $\eta_{\text {inj }} \leq \eta_{\mathrm{TP}}$, the temperature and density of the thermal gas remains constant. For the example shown in Fig. 1, $\eta_{\mathrm{TP}} \simeq 2.5 \times 10^{-6}$.

In contrast, the energetic portions of NL spectra are set by the conservation of mass, momentum, and energy fluxes and any change in the relativistic population influences the thermal population. When acceleration is efficient, the relation between the shock velocity, $u_{0}$, and the temperature of the shocked gas, $T_{2}$, is no longer approximated by the commonly used expression for a strong, TP shock. That is,

$\frac{k T_{2}}{\mu m_{\mathrm{p}} u_{0}^{2}} \neq \frac{3}{16}$,

where $\mu m_{\mathrm{p}}$ is the mean particle mass and $k$ is Boltzmann's constant. If the equality in Eq. (4) is assumed for a fully ionized plasma with $n_{\mathrm{He}} / n_{\mathrm{p} 0}=0.1$ ( $n_{\mathrm{He}}$ is the unshocked number density of helium) and equal downstream temperatures for all species,

$T_{2} \simeq 1.3 \times 10^{7}\left(\frac{u_{0}}{10^{3} \mathrm{~km} \mathrm{~s}^{-1}}\right)^{2} \mathrm{~K}$.

For young SNRs, this predicts temperatures just behind the blast wave well above those capable of explaining observed $\mathrm{X}$-ray line emission (e.g., $u_{0}=3000 \mathrm{~km} \mathrm{~s}^{-1} \rightarrow T_{2} \simeq 1.2 \times$ $10^{8} \mathrm{~K}$ ) and suggests that the shocked electron temperature is considerably less than predicted by Eq. (5) (e.g., Hughes et al. 2000; Hwang et al. 2002; Long et al. 2003).

Normally, it is assumed that electrons obtain a much lower temperature than the ions in the shock layer and take some time to equilibrate downstream through Coulomb collisions (see Decourchelle \& Ellison 2001, for a discussion of electron-ion equilibration in modified shocks). However, the heating process in the shock layer is certainly dominated by collisionless, wave-particle interactions which are poorly understood. The simplest possibility, that all particles upon crossing the shock gain a speed differential $\Delta u \sim u_{0}-u_{2}$, where $u_{2}$ is the downstream flow speed as seen from the shock, predicts that ions are heated far more than electrons ${ }^{5}$. Nevertheless, the possibility

\footnotetext{
5 That the situation is clearly more complicated than this is evident from the fact that if both species only receive $\Delta u$ upon crossing the shock, energy is not conserved (see Jones \& Ellison 1987).
} 
exists that electrons equilibrate faster than this simple conjecture implies and the fact that efficient acceleration produces a considerably lower proton temperature (Fig. 1) in strong shocks than the TP prediction of Eq. (5), suggests that it may not be as obvious that $T_{\mathrm{e} 2} / T_{\mathrm{p} 2} \ll 1$ immediately behind the shock 6 .

Furthermore, radio synchrotron observations have long confirmed that many SNRs accelerate electrons to relativistic energies, although it is not certain that these electrons are drawn from the shock heated population in all cases. What is certain is that if relatively cold upstream electrons enter the downstream region without being heated and then slowly equilibrate as they advect away downstream, very few if any will be injected into the Fermi acceleration process. In the simplest, kinematic models of injection in Fermi acceleration (e.g., Jones \& Ellison 1991; Baring et al. 1999), the efficient acceleration of thermal electrons is inconsistent with a lack of electron heating in the shock layer.

\subsection{CR-hydrodynamic coupling}

When efficient particle acceleration shifts energy from the thermal gas into relativistic particles, the fraction of total energy density in pressure is lowered and the evolution of the SNR is modified. The modified evolution, in turn, modifies the shock parameters which determine the acceleration. We model this coupling by including nonlinear shock acceleration in a standard 1D hydrodynamic simulation as follows.

Briefly: (i) the hydro simulation is initiated at some time, $t_{\text {start }}<0.1 t_{\text {ch }}$, after the supernova explosion with a plateau-power-law ejecta distribution and a constant interstellar medium density, $\rho_{\text {ISM }}$; (ii) at each time step, the hydro evolves and the radii and Mach numbers of the forward and reverse shocks are determined; (iii) with this shock information, the $\mathrm{CR}$ acceleration is calculated using the approximate model of Ellison et al. (2000) with the injection parameter, $\eta_{\text {inj }}$, kept constant during the simulation and having the same value at the forward and reverse shocks ${ }^{7}$; (iv) The acceleration calculation provides the overall compression ratio, $r_{\text {tot }}$, and the effective ratio of specific heats is determined by

$\gamma_{\mathrm{eff}}=\frac{M_{\mathrm{S}}^{2}\left(r_{\mathrm{tot}}+1\right)-2 r_{\mathrm{tot}}}{M_{\mathrm{S}}^{2}\left(r_{\mathrm{tot}}-1\right)}$,

${ }^{6}$ Estimates of $T_{\mathrm{e} 2} / T_{\mathrm{p} 2}$ based on optical and UV line observations have been obtained just behind a few SNR shocks (e.g., Raymond et al. 1995; Laming et al. 1996; Ghavamian 1999). These show a wide range, i.e., $0.2<T_{\mathrm{e} 2} / T_{\mathrm{p} 2}<0.8$, with large uncertainties and are generally restricted to regions that are partially neutral where the most efficient particle acceleration may not be occurring (see Drury et al. 2001, for a more complete discussion).

${ }^{7}$ In an actual SNR, of course, the injection efficiency might vary with time, vary over the shock surface, or be different at the forward and reverse shocks (as in our model of Kepler's SNR; Decourchelle et al. 2000). For the general results presented here, keeping $\eta_{\text {inj }}$ constant avoids unnecessary complexity. It is important to note, however, that the acceleration efficiency, i.e., the fraction of ram kinetic energy that is transferred to superthermal particles, depends on the shock parameters (Mach numbers, shock age, etc.) as well as on $\eta_{\text {inj }}$ and does vary with age and between the forward and reverse shocks even if $\eta_{\text {inj }}$ is constant. where we note that $\gamma_{\mathrm{eff}}<\left(P / \rho_{\mathrm{en}}\right)+1$ since $\gamma_{\mathrm{eff}}$ includes the effects from "escaping" particles ${ }^{8}$. Here, $P\left(\rho_{\text {en }}\right)$ is the pressure (energy density) of the shocked gas; (v) The coupling between the CR acceleration and the hydrodynamics is accomplished by replacing the ratio of specific heats used in the hydrodynamic equations with $\gamma_{\text {eff }}$ in the shells spanning the shock transition. We use a Lagrangian mode in the hydro simulation so the simulation grid moves with the mass. Once a mass element has been shocked and a $\gamma_{\text {eff }}$ has been assigned to it using Eq. (6), that mass element retains that $\gamma_{\mathrm{eff}}$ for the rest of the simulation or until it reencounters a shock. Even though energy is leaving the system via the highest energy cosmic rays, we do not explicitly remove energy from the hydro. Lowering $\gamma_{\mathrm{eff}}$ causes the gas to be more compressible and mimics the escaping energy in one step; (vi) as the SNR evolves, the proton distribution functions in momentum, $f(p)$, associated with each shocked shell undergo adiabatic losses; and finally, (vii) at the end of the simulation, the $f(p)$ 's from all of the shells are summed to determine the total contribution to the Galactic CR source population.

To start, we show in Fig. 2 a TP case where $\gamma_{\text {eff }}=5 / 3$ always (top panel). For this example and all others presented in this paper, we have taken $E_{\mathrm{sn}}=3 \times 10^{51} \mathrm{erg}, M_{\mathrm{ej}}=1.4 M_{\odot}$, $B_{0}=20 \mu \mathrm{G}$, and a constant density ISM with $n_{\mathrm{p} 0}=0.3 \mathrm{~cm}^{-3}$, and $f_{\mathrm{He}}=0.1$. These values give a characteristic age, $t_{\mathrm{ch}}=$ $R_{\mathrm{ch}} / V_{\mathrm{ch}} \simeq 210 \mathrm{yr}$. The initial ejecta density distribution is a power law $\left(\rho \propto r^{-7}\right)$ with a flat plateau at small radii. The simulation spans $6000 \mathrm{yr}$ and various quantities are shown, as a function of radius, at different times during the SNR evolution. The temperature shown in panel (b) is the average temperature defined as

$<T_{2}>=\frac{P}{\rho} \frac{\mu m_{\mathrm{p}}}{k} \times f_{\mathrm{th}}$,

where $P$ is the pressure, $\rho$ is the density, $\mu=\left(1+4 f_{\mathrm{He}}\right) /(2+$ $\left.3 f_{\mathrm{He}}\right), f_{\mathrm{He}}=0.1$ is the ratio of helium atoms to protons, and $f_{\mathrm{th}}$ is the fraction of pressure in thermal particles. In this plot and all others, we assume the plasma to be fully ionized. When acceleration occurs, superthermal particles contribute to the total hydrodynamic pressure, $P$. We define the temperature from that fraction of total pressure in the "thermal" particles, $f_{\text {th }}$. This fraction is determined from $f(p)$ (see Fig. 1 where the division between thermal and superthermal particles is indicated).

For the particular parameters assumed for the model shown in Fig. 2, the SNR is still in the self-similar phase after $100 \mathrm{yr}$ as the reverse shock moves through the power-law portion of the ejecta. By 300 years, the reverse shock is moving through the plateau region of the ejecta and the forward shock has reached $R_{\mathrm{FS}} \simeq 4$ pc. The density of the unshocked ejecta is now well below the ambient ISM density $\rho_{\mathrm{ISM}}=1.4 m_{\mathrm{p}} n_{\mathrm{p} 0}$ at this stage. By 1000 years, $R_{\mathrm{FS}} \sim 8 \mathrm{pc}$, the RS is now moving back toward the origin, and the energy of the explosion is shifting from being in the kinetic energy of the ejecta to thermal pressure.

\footnotetext{
${ }^{8}$ Performing the acceleration calculation at each time step is computationally expensive. If the full spectrum is not needed and only efficient acceleration is considered, a faster approximation can use $r_{\text {tot }}=1.3 M_{\mathrm{A}}^{3 / 8}$ if $M_{\mathrm{A}}<M_{\mathrm{s}}^{2}$ or $r_{\mathrm{tot}}=1.5 M_{\mathrm{s}}^{3 / 4}$ otherwise (see Berezhko \& Ellison 1999).
} 


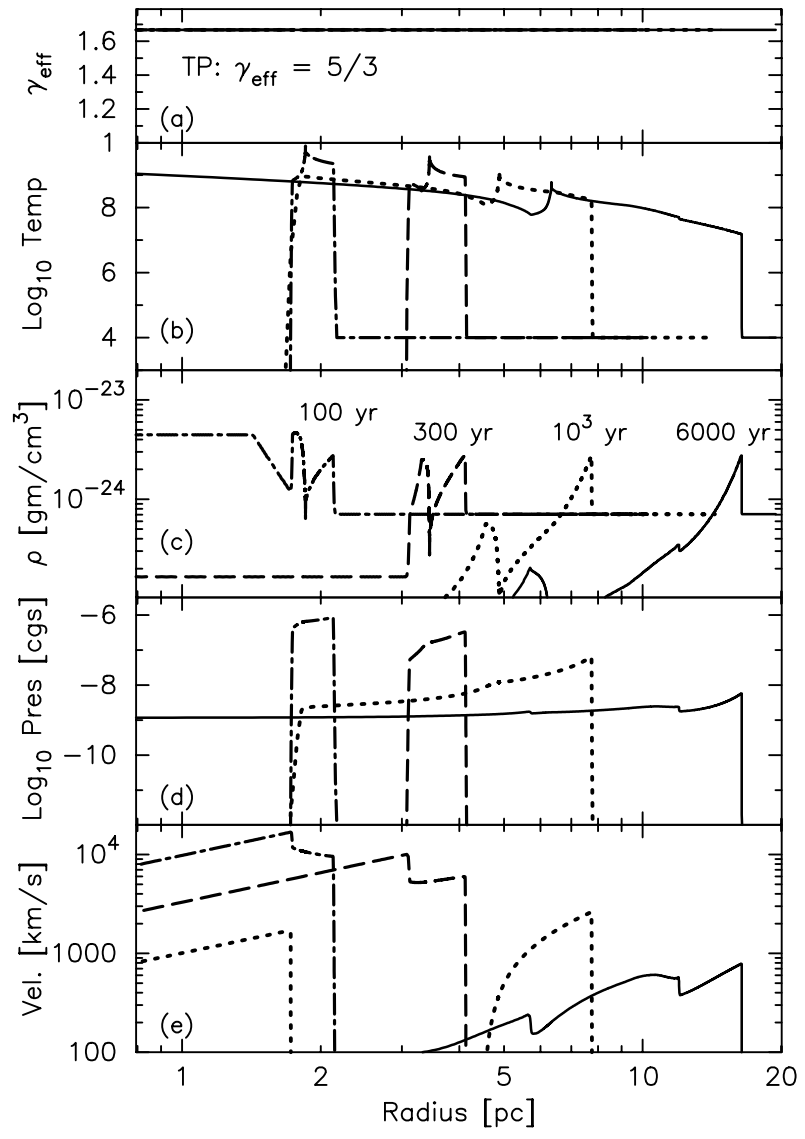

Fig. 2. Parameters from the hydro simulation versus radius at various times during the simulation as labeled. These are test-particle results with no adjustment of $\gamma_{\text {eff }}$ from particle acceleration. We have assumed $E_{\mathrm{sn}}=3 \times 10^{51} \mathrm{erg}, M_{\mathrm{ej}}=1.4 M_{\odot}$, and a constant density ISM with $n_{\mathrm{p} 0}=0.3 \mathrm{~cm}^{-3}$, and $f_{\mathrm{He}}=0.1$. The ejecta density structure is initially a power-law with index $n=7$ beyond the plateau at small radii (panel c)).

By 6000 years, the RS has collapsed back through the ejecta to the origin and the SNR is well into the Sedov phase with a FS speed of about (4/3)750 $\mathrm{km} \mathrm{s}^{-19}$.

In Fig. 3 we illustrate how particle acceleration influences the SNR shock evolution by comparing TP results $\left(\eta_{\text {inj }} \lesssim 10^{-6}\right)$ against a simulation with $\eta_{\text {inj }}=10^{-3}$. The figure shows the forward and reverse shock radii versus remnant age, $t_{\mathrm{snr}}$, for the same parameters as used in Fig. $2^{10}$. The TP results, i.e., those with $\gamma_{\mathrm{eff}}=5 / 3$ everywhere, are shown with solid curves and the NL results are shown with dashed curves. The dotted curve is the TP analytic approximation given by Truelove \& McKee (1999) for the FS and this matches the hydro results well over the entire time period. When NL effects are

\footnotetext{
${ }^{9}$ Note that the speed shown in the bottom panel of Fig. 2 and panel (d) of Fig. 4 is the flow speed in the frame of the explosion. The shock speed is given by $u_{0}=\left(r_{\mathrm{tot}} u_{\mathrm{ds}}-u_{\mathrm{up}}\right) /\left(r_{\mathrm{tot}}-1\right)$, where $u_{\mathrm{up}}$ $\left(u_{\mathrm{ds}}\right)$ is the flow speed just upstream (downstream) from the shock and $r_{\text {tot }}$ is the shock compression ratio. For the ISM conditions we assume here, $u_{\text {up }}$ always equals zero for the forward shock. At $t_{\text {snr }}=6000 \mathrm{yr}$ in Fig. 2 at the FS, $u_{\mathrm{ds}} \simeq 750 \mathrm{~km} \mathrm{~s}^{-1}, r_{\mathrm{tot}} \simeq 4$, and $u_{0} \simeq 1000 \mathrm{~km} \mathrm{~s}^{-1}$. ${ }^{10}$ We do not follow particle acceleration at the reverse shock after it first collapses to zero radius.
}

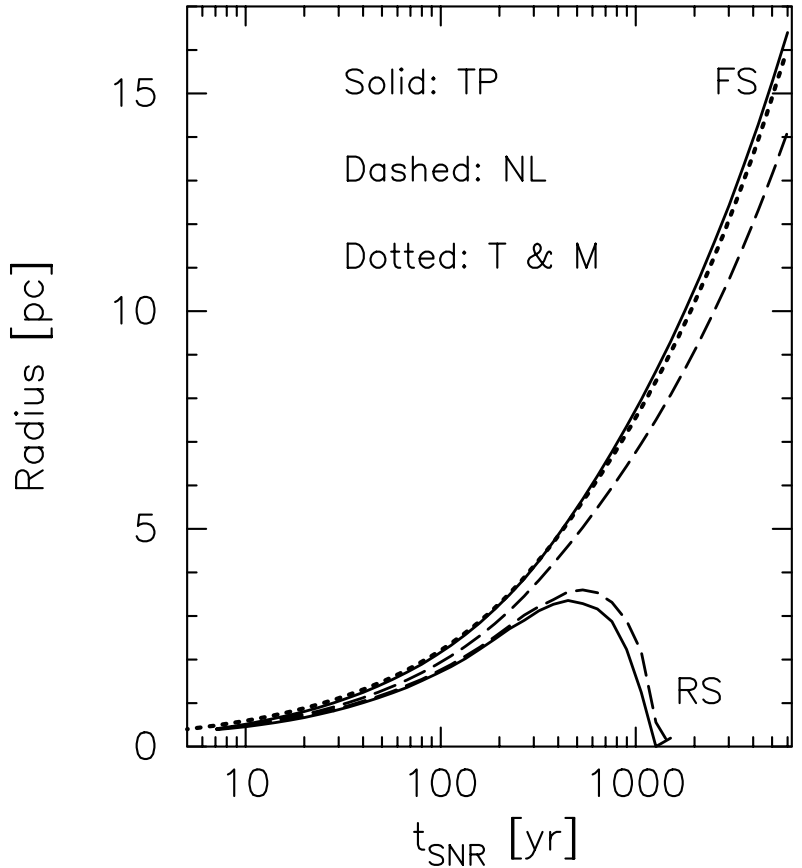

Fig. 3. Shock radius versus SNR age for forward (FS) and reverse shocks (RS) obtained with our hydrodynamic simulation. The dashed curves show NL results with efficient particle acceleration $\left(\eta_{\text {inj }}=\right.$ $10^{-3}$ ), while the solid curves are test-particle (TP) shocks with no particle acceleration. The dotted curve shows the TP forward shock radius obtained with the approximate analytic expression given in Truelove $\&$ McKee (1999).

important, the region between the forward and reverse shocks becomes narrower and denser, a result fully consistent with the analytic results of Decourchelle et al. (2000) and the multidimensional hydro simulations of Blondin \& Ellison (2001).

In Fig. 4 we compare TP profiles to NL ones at $t_{\mathrm{snr}}=200$ and $1000 \mathrm{yr}$. In all panels, the heavy-weight solid curves are the TP results $\left(\eta_{\text {inj }} \lesssim 10^{-6}\right)$, the dotted curves have $\eta_{\text {inj }}=$ $1.2 \times 10^{-4}$, and the dashed curves have $\eta_{\text {inj }}=10^{-3}$. Panels (a) and (e) show the reduction in $\gamma_{\text {eff }}$ that occurs as shock acceleration shifts energy into relativistic particles. This produces compression ratios greater than 4 and results in denser, narrower regions between the forward and reverse shocks as shown in the density profiles (b) and (f). The separation between the forward and reverse shocks varies considerably with $t_{\mathrm{snr}}$ and $\eta_{\text {inj. }}$. At $t_{\mathrm{snr}}=200 \mathrm{yr}$, this separation is $\sim 0.25 \mathrm{pc}$ for $\eta_{\mathrm{inj}}=10^{-3}$ and $\sim 0.7 \mathrm{pc}$ for the TP case, while at $t_{\mathrm{snr}}=1000 \mathrm{yr}$ it is $\sim 4.2 \mathrm{pc}$ for $\eta_{\text {inj }}=10^{-3}$ and $\sim 6 \mathrm{pc}$ for the TP case. The shift in energy out of the thermal gas also results in a significant decrease in the temperature of the shocked gas in the NL case, as shown in panels (c) and (g).

The flow speed is shown in the bottom panel and the forward and reverse shocks are easily identified in all panels. At $t_{\mathrm{snr}}=1000 \mathrm{yr}$, the flow speeds just behind the forward shocks of the three examples are about equal, i.e., $u_{\mathrm{ds}}(\mathrm{TP}) \simeq$ $2600 \mathrm{~km} \mathrm{~s}^{-1}$ and $u_{\mathrm{ds}}\left(\eta_{\text {inj }}=10^{-3}\right) \simeq 2500 \mathrm{~km} \mathrm{~s}^{-1}$. However, since the shock speed depends on $r_{\text {tot }}$, the TP shock is moving faster, i.e., $u_{0}(\mathrm{TP}) \simeq(4 / 3) 2600 \simeq 3500 \mathrm{~km} \mathrm{~s}^{-1}$, while $u_{0}\left(\eta_{\text {inj }}=10^{-3}\right) \simeq(7 / 6) 2500 \simeq 2900 \mathrm{~km} \mathrm{~s}^{-1}$. 


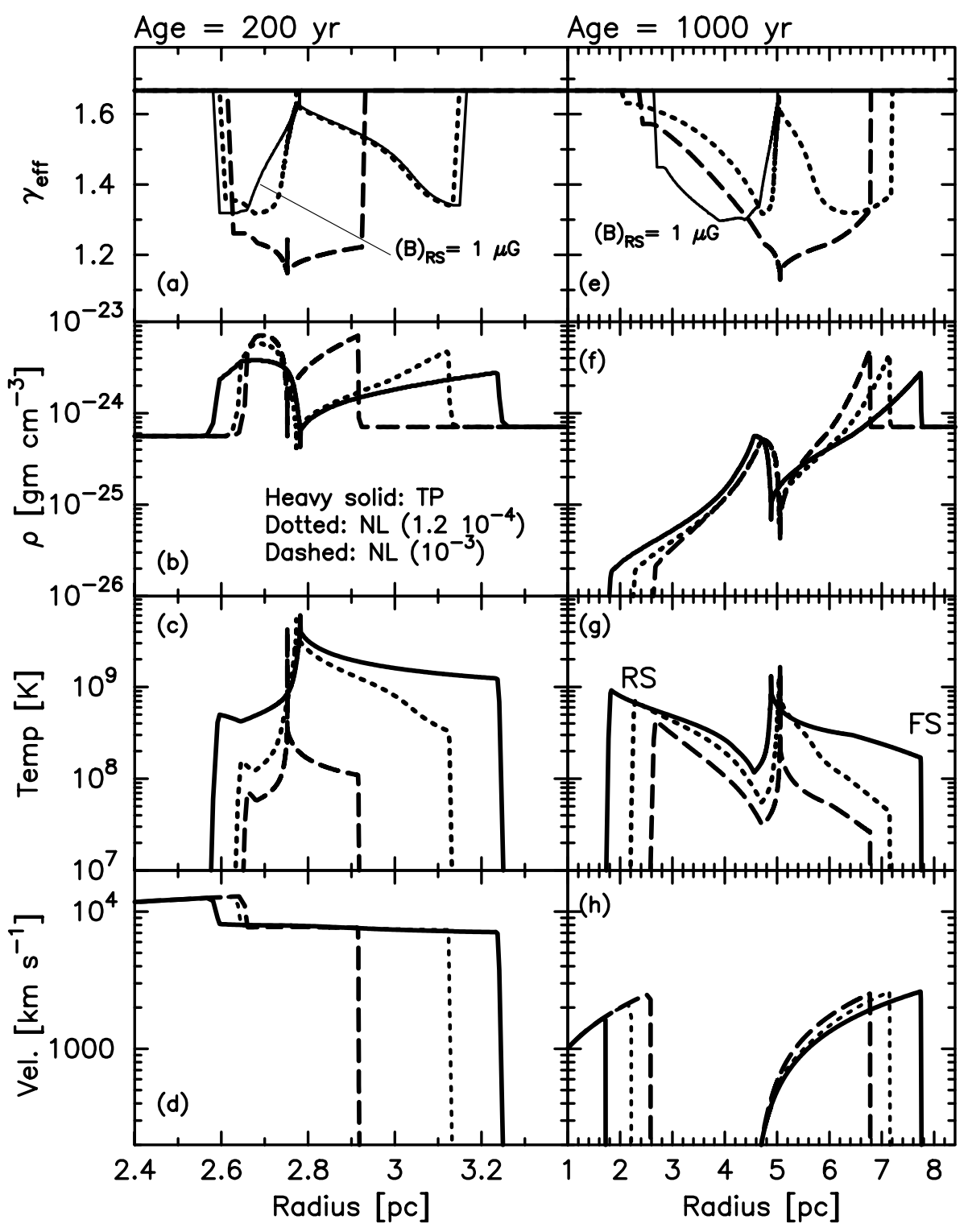

Fig. 4. Comparison of the parameters for a test-particle simulation (solid curves) with those undergoing particle acceleration. The results are shown at $t_{\mathrm{snr}}=200$ and $1000 \mathrm{yr}$ and the supernova parameters are the same as those in Fig. 2. The light-weight solid curves in panels a) and e) show the effect on $\gamma_{\mathrm{eff}}$ of reducing the unshocked ejecta magnetic field from $\left(B_{0}\right)_{\mathrm{RS}}=20 \mu \mathrm{G}$ to $1 \mu \mathrm{G}$. Apart from $\left(B_{0}\right)_{\mathrm{RS}}$, all other parameters are the same as in the $\eta_{\mathrm{inj}}=1.2 \times 10^{-4}$ examples shown with dotted curves. In panel $\left.\mathbf{e}\right)$, the shocked ISM portion of the $\left(B_{0}\right)_{\mathrm{RS}}=1 \mu \mathrm{G}$ curve is essentially identical to the dotted curve.

The intermediate NL case with $\eta_{\text {inj }}=1.2 \times 10^{-4}$ (dotted curves) produces temperatures and compression ratios between the TP and $\eta_{\text {inj }}=10^{-3}$ cases, as expected. However, as seen in the variations of $\gamma_{\text {eff }}$ (panels (a) and (e) of Fig. 4), the $\eta_{\text {inj }}=1.2 \times 10^{-4}$ case acts quite differently at the beginning of the simulation. As described in Berezhko \& Ellison (1999), unmodified shocks with $r_{\text {tot }} \simeq 4$ can occur for high Mach numbers if $\eta_{\text {inj }}$ is small enough. In this case, the pressure in relativistic particles, $P_{\text {rel }}$, is small compared to $\rho_{0} u_{0}^{2}$ and these particles do not slow the incoming gas enough to produce the nonlinear shock modification. For a given $\eta_{\text {inj }}$, as the SNR ages, $u_{0}$ decreases, $P_{\text {rel }} /\left(\rho_{0} u_{0}^{2}\right)$ increases, and the shock acceleration becomes more efficient and more nonlinear. Thus, for relatively inefficient injection, $\gamma_{\text {eff }}$ drops and $r_{\text {tot }}$ increases in the early stages of evolution, exactly the opposite behavior as with efficient injection ${ }^{11}$.

After the initial transition from a strong, unmodified shock to a strong, modified one, the forward shock will enter the long stage where it slowly weakens, the acceleration becomes less efficient, and $\gamma_{\mathrm{eff}}$ increases toward 5/3. However, as shown by the dotted curve in panel (e) in Fig. 4, the reverse shock weakens much more quickly and at $t_{\mathrm{snr}}=1000 \mathrm{yr}$ has $\gamma_{\mathrm{eff}} \simeq 5 / 3$. Since we assume that $B_{0}$ is a constant everywhere, the RS

\footnotetext{
${ }^{11}$ As discussed in Berezhko \& Ellison (1999), it is by no means certain that actual SNR shocks will have injection rates low enough for high Mach number, unmodified shocks to occur. Furthermore, there may be large differences in injection rates for parallel and oblique regions of the same SNR blast wave, making it possible that some regions are highly modified and others unmodified.
} 


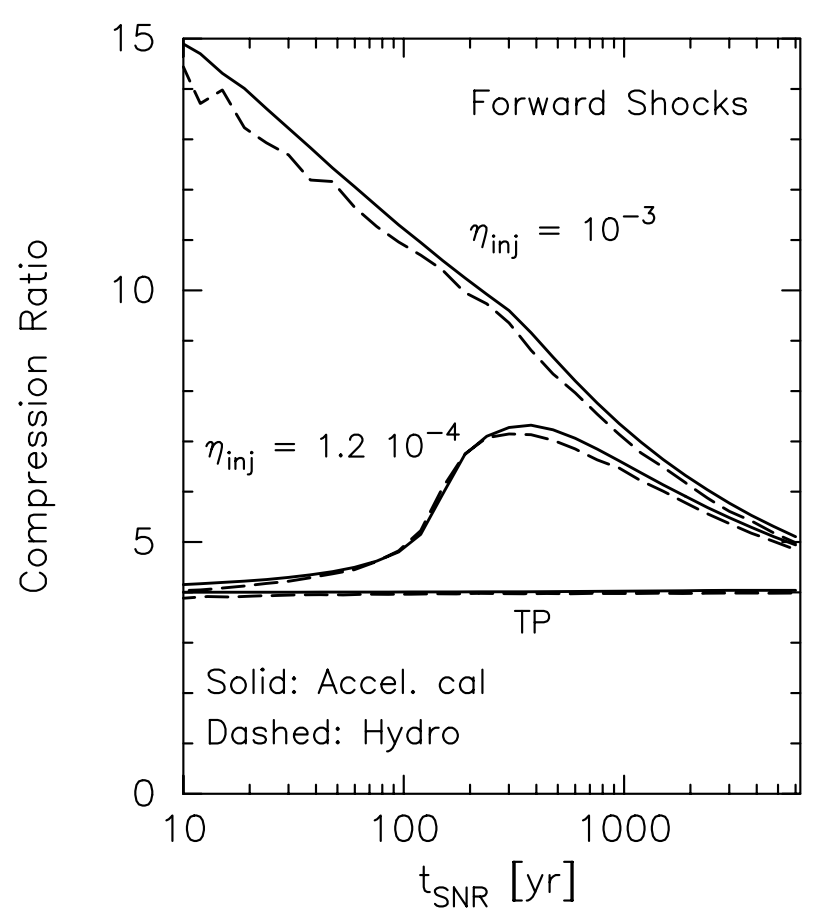

Fig. 5. Forward shock compression ratios vs. supernova age for various injection rates, $\eta_{\text {inj. }}$. In all cases, the solid curves are obtained from the NL particle acceleration calculation and the dashed curves are obtained from the density ratio, $\rho_{\mathrm{dw}} / \rho_{\mathrm{up}}$, just downstream and upstream from the shock.

weakens as the ejecta density drops and the magnetic pressure becomes dominant over the gas pressure. In an actual SNR, magnetic flux conservation in the expanding, unshocked ejecta would cause $B_{0}$ to weaken as the ejecta density drops and the RS would remain stronger than with a constant $B_{0}$. For a given unshocked density, a weak $B_{0}$ results in efficient shock acceleration because the Alfvén Mach number increases with decreasing $B_{0}$, and because the amount of energy transferred out of accelerated particles into waves and then into heating of the precursor gets less. Of course, if the magnetic field gets too weak, the gyroradii of accelerated particles will become comparable to the shock radius, or the acceleration time will become comparable to $t_{\mathrm{snr}}$, before high particle energies or high efficiencies are obtained. To illustrate the effects of a low ejecta magnetic field, we show in panels (a) and (e) of Fig. $4 \gamma_{\text {eff }}$ produced by our $\eta_{\text {inj }}=1.2 \times 10^{-4}$ example with the unshocked ejecta field set at $1 \mu \mathrm{G}$ (light-weight solid curves). As seen clearly in panel (e), $\gamma_{\text {eff }}$ remains lower at the end of the simulation than in the $\left(B_{0}\right)_{\mathrm{RS}}=20 \mu \mathrm{G}$ case, indicating more efficient acceleration.

In Fig. 5 we plot the compression ratio, $r_{\text {tot }}$, versus $t_{\text {snr }}$ for our TP and two NL examples. There are two sets of curves because there are two ways of determining $r_{\text {tot }}$. The solid curves are values obtained from the particle acceleration calculation which are then used in Eq. (6) to calculate $\gamma_{\text {eff. }}$ The dashed curves are determined directly from the hydro density ratio taken just downstream and upstream from the shock, $\rho_{\mathrm{dw}} / \rho_{\text {up }}$. In principle, these should be identical but in practice differences occur, indicating the inherent errors in our $\gamma_{\text {eff }}$ technique. The largest average difference shown here with $\eta_{\text {inj }}=10^{-3}$

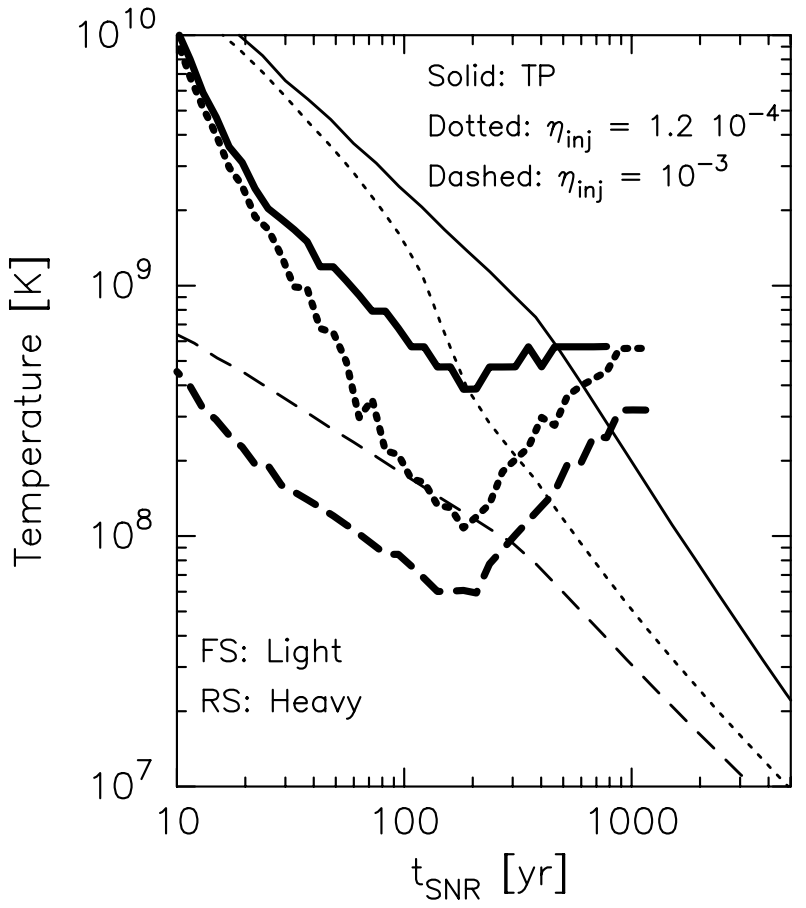

Fig. 6. Temperatures immediately behind the forward and reverse shocks as a function of $t_{\mathrm{snr}}$. The solid curves are the TP case, the dotted curves are for $\eta_{\mathrm{inj}}=1.2 \times 10^{-4}$, and the dashed curves are for $\eta_{\text {inj }}=10^{-3}$. The reverse shock temperatures are plotted with heavyweight curves.

is $<10 \%$. The $\eta_{\text {inj }}=1.2 \times 10^{-4}$ case clearly shows the rapid transition from the strong, unmodified shock with $r_{\text {tot }} \sim 4$ at early times to the modified shock with $r_{\text {tot }}>4$ at intermediate times. The striking difference between the $\eta_{\text {inj }}=10^{-3}$ and $\eta_{\text {inj }}=1.2 \times 10^{-4}$ cases will leave an imprint on the post-shock gas that may be an important diagnostic for determining if efficient Fermi acceleration takes place in young SNRs.

In Fig. 6 we show the postshock proton temperatures for the same three examples. Comparing the heavy-weight solid and dashed curves shows that the RS temperature in the $\eta_{\mathrm{inj}}=$ $10^{-3}$ efficient shock is less than $\sim 1 / 10$ that of the test-particle shock over most of the time period shown. This is a difference large enough to have profound consequences for the interpretation of X-ray thermal emission in young SNRs. Furthermore, the temperature in the intermediate case $\left(\eta_{\text {inj }}=1.2 \times 10^{-4}\right)$ shows a stronger time variation than either the TP or $\eta_{\text {inj }}=10^{-3}$ case. This is reflected as a somewhat steeper spatial gradient in temperature as seen in panel (g) of Fig. 4, again offering a possible diagnostic to test for efficient particle acceleration.

\subsubsection{Comparison with analytic model of Decourchelle et al.}

In our previous work (Decourchelle et al. 2000) we coupled NL acceleration with an analytic, self-similar description of the SNR hydrodynamics (i.e., Chevalier 1983) and a non-equilibrium ionization calculation of thermal X-ray emission. That model used the same algebraic acceleration calculation we use here with the same injection parameter, $\eta_{\text {inj }}$. 
The assumptions of the two models differ mainly in the way the hydrodynamics are treated.

While the hydro simulation has a one-fluid approach with an effective ratio of specific heats derived from the compression ratio (Eq. (6)), the analytical, self-similar solutions are based on a two-fluid approach: a nonrelativistic one with $\gamma=5 / 3$ and a relativistic one with $\gamma=4 / 3$. The NL particle acceleration calculation provides the compression ratio and the fraction of total pressure in relativistic gas at both shocks which are used to determine the boundary conditions of the self-similar solution. Imposing the compression ratios, which can be greater than 7 , mimics the effects of particle loss. The limitations of the self-similar calculation are that the RS must be propagating in the power-law portion of the ejecta density profile, and that it assumes that the forward and reverse shock compression ratios, as well as the fractions of total pressure in relativistic gas, remain constant throughout the evolution. A limitation of our hydrodynamic simulation is that it assigns at the shocks to an element of gas an effective gamma, which is kept constant during its post-shock evolution.

In the top three panels of Fig. 7 we compare the density structures for our two models at $t_{\mathrm{snr}}=100 \mathrm{yr}$ when the RS is still propagating in the power-law portion of the ejecta density profile. We use the same supernova and ISM parameters as in our previous runs. For the TP (panel (a)) and $\eta_{\text {inj }}=10^{-3}$ (panel (b)) cases, the correspondence between the two models is extremely close with small differences resulting mainly from the finite grid used in the hydro simulation. The reason for this comes from the fact that self-similarity is satisfied in the TP case where $r_{\text {tot }} \simeq 4$ always. It is also approximately satisfied in the $\eta_{\text {inj }}=10^{-3}$ case since $r_{\text {tot }}$, and therefore $\gamma_{\mathrm{eff}}$, remains fairly constant during the $100 \mathrm{yr}$ evolution, as indicated by the dashed curve in panel (a) of Fig. 4.

While it was not obvious that the $\gamma_{\text {eff }}$ procedure used in the hydro model would be a good approximation, the excellent agreement we see with the analytic model, which only assumes self-similarity, justifies this approach, at least at these early times.

Much larger differences in the two models are seen in panels (c) and (d) where $\eta_{\text {inj }}=2 \times 10^{-4}$. In this case, $r_{\text {tot }}$ and therefore $\gamma_{\text {eff }}$ vary strongly during $100 \mathrm{yr}$ (similar to the dotted curve in panel (a) of Fig. 4), and self-similar conditions no longer apply. Since the analytic model takes $r_{\text {tot }}$ at the forward and reverse shocks at the final time and applies these values for the entire evolution, it is effectively using values, at least for the FS in the $\eta_{\text {inj }}=2 \times 10^{-4}$ case, which are considerably greater than the average values used in the hydro calculation. Using a larger $r_{\text {tot }}$ means there is less pressure for a given energy density pushing the shock in the self-similar model than in the hydro model. Therefore, the FS in the hydro calculation travels faster and extends further than in the analytic calculation. The variation in $\gamma_{\mathrm{eff}}$ is less in the shocked ejecta (again similar to the dotted curve in panel (a) of Fig. 4) so the difference in the two models is less there as well.

We consider the close correspondence between these two models in the TP and $\eta_{\text {inj }}=10^{-3}$ cases to be a clear indication that both adequately model the SNR hydrodynamics during self-similar conditions. The validation of the $\gamma_{\mathrm{eff}}$

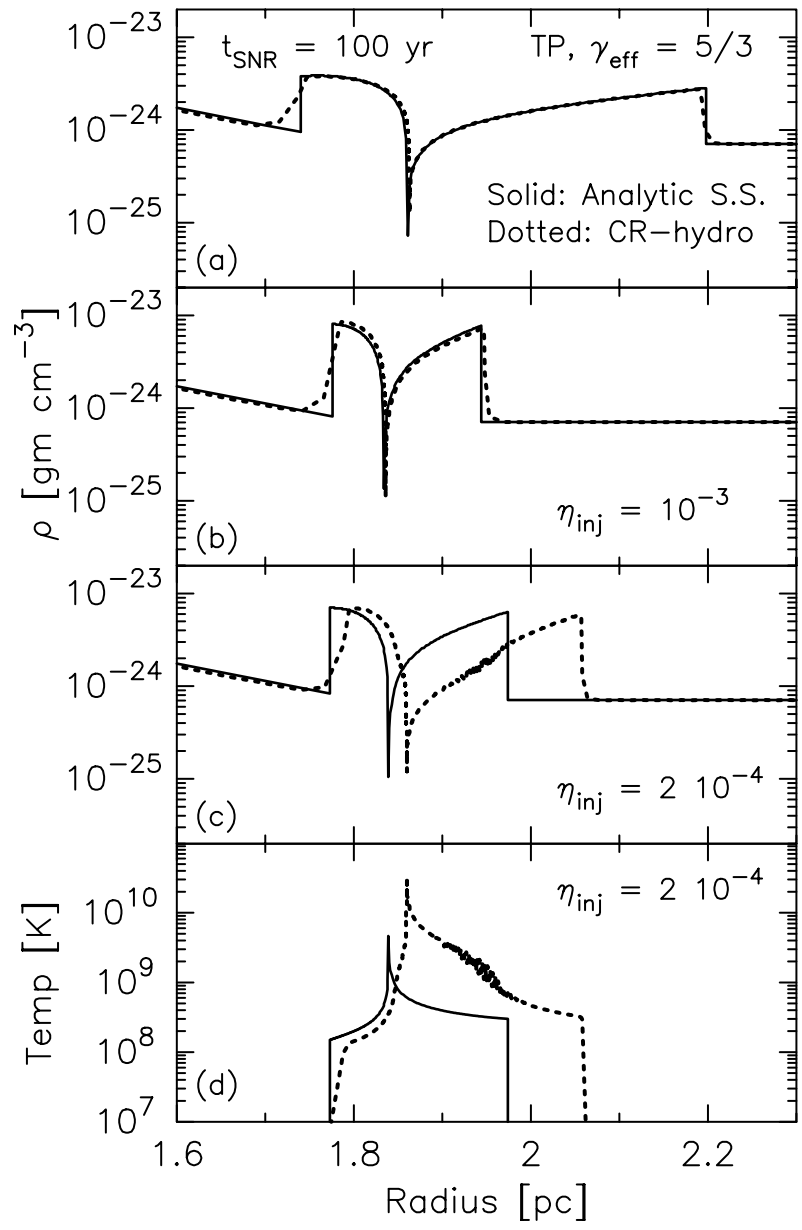

Fig. 7. Density (top three panels) versus radius for two NL models and a TP model, as indicated, at $t_{\mathrm{snr}}=100 \mathrm{yr}$. The bottom panel shows temperature versus radius for $\eta_{\text {inj }}=2 \times 10^{-4}$. In all panels, the solid curves are calculated using the analytic, self-similar model of Decourchelle et al. (2000) and the dotted curves are from the CR-hydro model presented here. In the CR-hydro model, some numerical noise is evident in the shocked ISM profiles for $\eta_{\text {inj }}=2 \times 10^{-4}$.

approach by our two-fluid description of the post-shock flow in the framework of self-similar, cosmic-ray modified Chevalier solutions means the more general hydro simulation can be used with some confidence when self-similar conditions do not apply. This will also allow us to use the analytic model to test the hydro model when X-ray thermal and broad-band photon emission is included.

\subsection{Energetic proton spectra}

The proton distribution functions that are calculated at the forward and reverse shocks during the SNR evolution can be summed to determine the total contribution to the cosmic-ray spectrum. The amount of material swept up by each shock is used to weight each spectrum and convert $f(p)$ to $N(p, \Delta t)$ where $4 \pi \int_{0}^{\infty} p^{2} N(p, \Delta t) \mathrm{d} p$ is the total number of particles overtaken by the shock in the time interval, $\Delta t$. The $N(p, \Delta t)$ 's are then added together, with each one adjusted for adiabatic losses during the time from when the spectrum was calculated 
until the end of the simulation (e.g., Reynolds 1998), to produce $N(p)^{12}$. Typical proton spectra calculated with $B_{0}=$ $20 \mu \mathrm{G}$ at $t_{\mathrm{snr}}=500 \mathrm{yr}\left(t_{\mathrm{snr}} / t_{\mathrm{ch}} \simeq 2.4\right.$ for $E_{\mathrm{sn}}=3 \times 10^{51} \mathrm{erg}$, $M_{\mathrm{ej}}=1.4 M_{\odot}, n_{\mathrm{p} 0}=0.3 \mathrm{~cm}^{-3}$, and $\left.f_{\mathrm{He}}=0.1\right)$ are shown in Fig. 8. The NL examples produce cosmic rays at the expense of heating and show lower temperatures, as indicated by the positions of the thermal peaks. The dashed curve is the result with the most efficient injection $\left(\eta_{\text {inj }}=10^{-3}\right)$ and this spectrum is the flattest at relativistic energies (before the turnover at $p_{\max }$ ). The spectrum with $\eta_{\text {inj }}=1.2 \times 10^{-4}$ is the most distorted from a Maxwellian at thermal energies due to the summing of spectra which evolve from unmodified $\left(r_{\text {tot }} \simeq 4\right.$ ) to strongly modified $\left(r_{\text {tot }}>4\right)$, as discussed above.

To illustrate the effects of a weakening unshocked ejecta magnetic field, we show in the bottom panel of Fig. 8 (thin solid curve) $N(p)$ for the case where the unshocked ejecta field is set at $\left(B_{0}\right)_{\mathrm{RS}}=1 \mu \mathrm{G}$, a factor of 20 lower than in the other cases. The unshocked ISM field is kept at $20 \mu \mathrm{G}$ and $\eta_{\text {inj }}=1.2 \times 10^{-4}$ for both the forward and reverse shocks. The flatness of the superthermal part of the spectrum compared to the dotted curve indicates that the RS is stronger (i.e., $r_{\text {tot }}$ is larger) than when $\left(B_{0}\right)_{\mathrm{RS}}=20 \mu \mathrm{G}$ (see Fig. 4). However, $p_{\max }$ is well below that in the $\left(B_{0}\right)_{\mathrm{RS}}=20 \mu \mathrm{G}$ cases because the weak field produces large gyroradii and high momentum particles cannot be accelerated. The FS spectrum is only slightly changed by changing $\left(B_{0}\right)_{\mathrm{RS}}$ and is not shown.

In Fig. 9 we show spectra at 1000,6000 , and $4 \times 10^{4} \mathrm{yr}$ for the same ISM and SN parameters with $\eta_{\text {inj }}=10^{-3}$. As the FS expands and weakens, the acceleration efficiency drops and the relativistic portion of the spectrum steepens. As expected, particles accelerated at the RS decrease in importance in later stages of the SNR. As we show in Fig. 11 below, even moderately efficient injection (i.e., $\eta_{\text {inj }} \gtrsim 10^{-4}$ ) results in approximately $50 \%$ of the explosion energy being put into relativistic particles over the lifetime of the SNR. Another important property shown in the top panel of Fig. 9 is that, even with $\eta_{\text {inj }}=10^{-3}$ which produces strongly curved spectra at early times, the total particle distribution function after $4 \times 10^{4} \mathrm{yr}$ is approximately $N(p) \propto p^{-2}$ at relativistic energies. Thus the total cosmic-ray production is fairly consistent with the observed CR flux after energy dependent escape from the galaxy is included.

\subsection{Comparison with kinetic models}

One of the most complete models of nonlinear CR production in SNRs is that of Berezhko and co-workers (e.g., Berezhko et al. 1996; Berezhko \& Völk 1997) where the timedependent CR transport equations are solved together with the gas-dynamic equations in spherical symmetry. This model gives the radial distribution of gas and accelerated CR spectrum at any phase of the SNR evolution and has been used with good success to model a number of young SNRs including SN1006 (Berezhko et al. 2002), Cassiopeia A (Berezhko et al. 2003), and Tycho (Völk et al. 2002). In all of the above cases,

\footnotetext{
12 We do not include radiation (i.e., synchrotron) losses here since they are always negligible for ions. For electrons, of course, these losses must be considered.
}

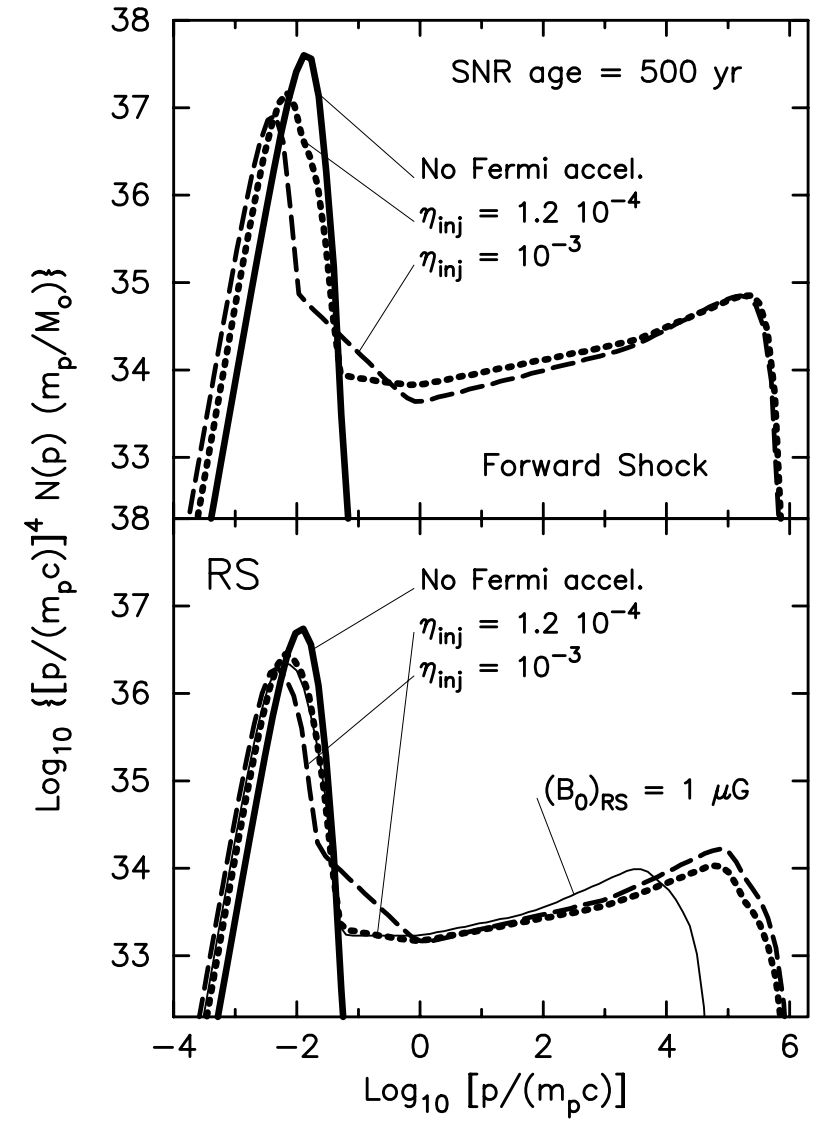

Fig. 8. Proton number distributions, $N(p)$ (multiplied by $\left.\left[p /\left(m_{\mathrm{p}} c\right)\right]^{4} m_{\mathrm{p}} / M_{\odot}\right)$, for hydro simulations with no acceleration (heavy solid curves), with $\eta_{\text {inj }}=1.2 \times 10^{-4}$ (dotted and thin solid curves), and with $\eta_{\text {inj }}=10^{-3}$ (dashed curves) summed over 500 years. The spectra in the top panel are from the shocked ISM (forward shock), while those in the bottom panel are from the shocked ejecta (reverse shock). The $N(p)$ 's are absolutely normalized to the total number of protons overtaken by the shock during their lifetime. All spectra have been adjusted for adiabatic expansion. The thin solid curve in the bottom panel shows the RS spectrum for the case where the unshocked ejecta magnetic field is set at $\left(B_{0}\right)_{\mathrm{RS}}=1 \mu \mathrm{G}$.

the authors conclude that satisfactory fits to the radio and $\mathrm{X}$ ray synchrotron emission, along with $\gamma$-rays where observed, are best obtained when the forward shocks are strongly modified by the efficient acceleration of protons in a high magnetic field. While similar results have been presented before (e.g., Reynolds \& Ellison 1992; Ellison et al. 2000, 2001), Berezhko's model probably contains the most complete physical description of the NL acceleration process at the FS, and the detailed modeling of particular SNRs clearly lends credibility to the suggestion that shocks in young SNRs can be strongly nonlinear with compression ratios greater than 4 .

Our CR-hydro model is complementary to that developed by Berezhko and co-workers in that we use a more approximate model of the NL acceleration, but have a more complete model of the hydrodynamics including the reverse shock. In particular, we do not follow the particles through the acceleration process where previously accelerated particles can continue to be re-accelerated at later times. Instead, we assume that all 


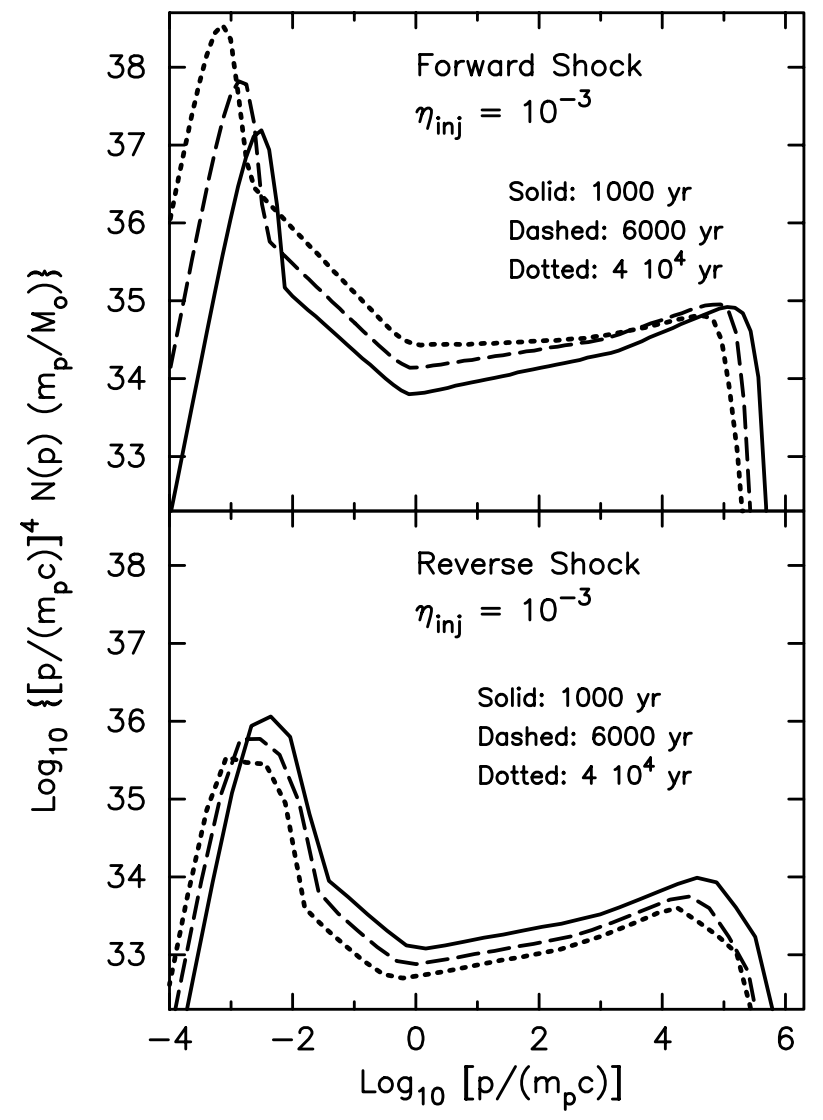

Fig. 9. Particle number distributions, $N(p)$ (multiplied by $\left.\left[p /\left(m_{\mathrm{p}} c\right)\right]^{4} m_{\mathrm{p}} / M_{\odot}\right)$ from the shocked ISM (FS) and shocked ejecta (RS) at various times during the SNR evolution. Efficient particle injection is assumed $\left(\eta_{\text {inj }}=10^{-3}\right)$ throughout the SNR evolution, but the fraction of energy in relativistic particles varies as the SNR ages. The acceleration efficiency decreases as the SNR ages and the shocks weaken. The RS contributes little to the total CR population at $t_{\mathrm{snr}}=4 \times 10^{4} \mathrm{yr}$. All spectra have been adjusted for adiabatic expansion.

accelerated particles originate as thermal particles in the upstream flow, are accelerated as the shock overtakes them, and then remain in the shell of material where they were accelerated, suffering adiabatic losses as the remnant ages. The re-acceleration of high energy particles with long diffusion lengths can be important as it adds to the maximum energy particles obtain (e.g. Berezhko et al. 1996), but this effect becomes less important as time goes on and new unshocked particles are overtaken and accelerated.

In Fig. 10 we compare our results with those of Berezhko et al. (2002) for the proton distribution at $t_{\mathrm{snr}}=1000 \mathrm{yr}$ they predict for SN1006. We have adjusted the normalization of the FS to obtain a good fit, but otherwise have used the environmental parameters given in Berezhko et al. While both our models require an arbitrary injection parameter defined as the fraction of thermal particles overtaken by the shock that become superthermal, the actual implementation of this parameter may be different in the two models. To fit SN 1006, Berezhko et al. use $\eta=2 \times 10^{-4}$; we have used $\eta_{\text {inj }}=1.2 \times 10^{-4}$ to match their results. A distinct advantage of Berezhko's model is that the maximum CR energy,

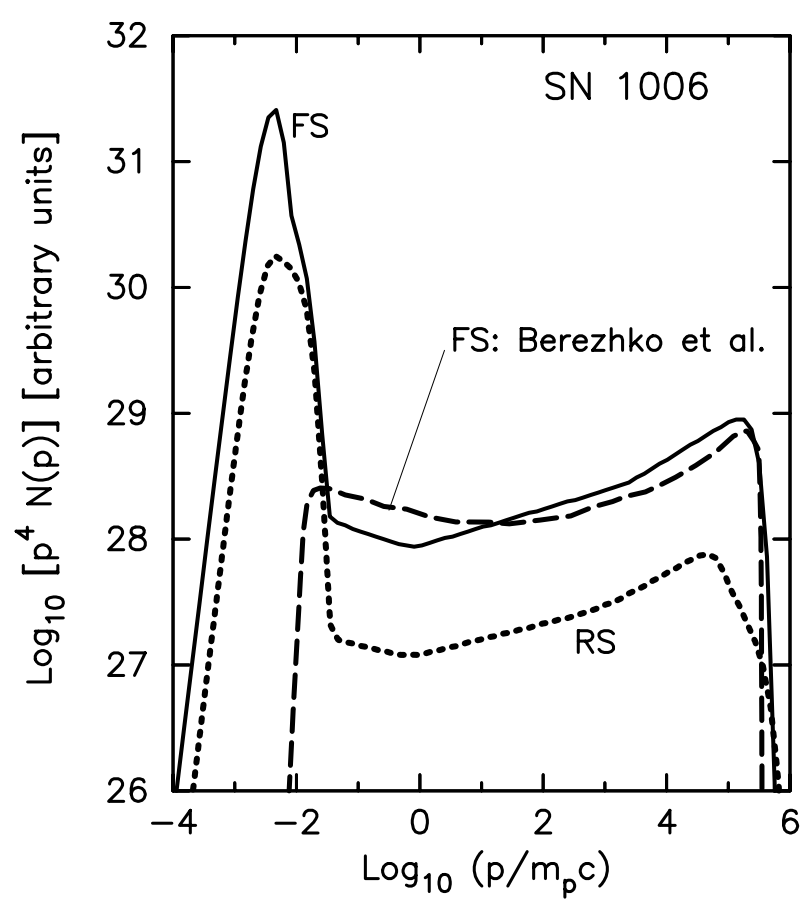

Fig. 10. The dashed curve is the inferred FS proton distribution of SN1006 as obtained by Berezhko et al. (2002) at $t_{\mathrm{snr}}=1000 \mathrm{yr}$. Using their input parameters for $E_{\mathrm{sn}}, M_{\mathrm{ej}}$, etc., we have matched this result (solid curve) with $\eta_{\text {inj }}=1.2 \times 10^{-4}, \alpha=4$, and $f_{\text {sk }}=0.1$ (see Eq. (2) and the discussion following it). The dotted curve is the corresponding proton distribution from shocked ejecta (RS) from our model.

$E_{\max }$, and the shape of $f(p)$ near $E_{\max }$ are determined selfconsistently. In our CR-hydro model, we parameterize these with $\alpha$ in Eq. (2) for the shape and with the fraction of shock radius, $f_{\mathrm{sk}}$, set equal to the maximum particle diffusion length to determine $E_{\max }$. To match the results given by Berezhko et al. (2002), we use $\alpha=4$ and $f_{\text {sk }}=0.1$. While fine tuning of our parameters could provide a more exact match than shown in Fig. 10, the particular values are unimportant compared to the fact that both models show a similar level of injection efficiency and shock modification. Considering the fundamental differences in the two models, the fit of superthermal particles accelerated by the FS shown in Fig. 10 is impressive. The concave shape, accentuated by plotting $p^{4} N(p)$, is very similar, although the piecewise nature of our approximation is apparent.

Equally important, the FS compression ratios are very similar, as shown in the top panel of Fig. 11 where the total and subshock compression ratios are compared for this SN1006 model (CR-hydro: solid curves; Berezhko et al.: dashed curves). As we discussed earlier, unmodified strong shocks can exist when $\eta_{\text {inj }}$ is relatively low and this is just what is predicted in both our models for $t_{\mathrm{snr}} \lesssim 200 \mathrm{yr}$ in SN 1006. As the FS slows, the acceleration becomes more efficient, $r_{\text {tot }}$ increases, and $r_{\text {sub }}$ starts to drop below 4 at $t_{\mathrm{snr}} \gtrsim 200 \mathrm{yr}$, indicating that the shocked proton temperature will be less than predicted in the TP case. The dotted curves are CR-hydro results with various $\eta_{\text {inj }}$ 's and show (curves $b$ and $c$ ) that the transition between unmodified and modified shocks can be extremely abrupt. For large enough $\eta_{\text {inj }}$ (curve $d$ ) unmodified solutions do not occur and $r_{\text {tot }}$ is large from the start. 
We take the excellent correspondence achieved with Berezhko's calculation as evidence that our CR-hydro model, with its algebraic NL particle acceleration approximation, is accurate.

As is clear in Fig. 10, Berezhko et al. (2002) do not treat thermal particles explicitly although, in principle, they could convert the thermal pressure and density into a Maxwellian distribution as we do. They also ignore the reverse shock, an approximation that is well justified if only continuum emission from relativistic particles is considered at times $t_{\mathrm{snr}} \gg t_{\mathrm{ch}}$. In order to predict thermal X-ray emission in young SNRs, however, thermal electrons must be modeled and particle heating and acceleration at the forward and reverse shocks must be selfconsistently determined. We do not show electron spectra here, but our NL acceleration model, with additional parameters, can produce these along with the broad-band continuum emission from radio to $\mathrm{TeV} \gamma$-rays produced by them (e.g., Ellison et al. 2001). The most important advantage our CR-hydro model has, however, is the ability to model the RS and the potential for including X-ray thermal emission from the shock-heated ejecta calculated using a non-equilibrium ionization calculation (e.g., Decourchelle et al. 2000).

In the bottom panel of Fig. 11 we show the fraction of explosion energy in relativistic particles, $E_{\mathrm{cr}} / E_{\mathrm{sn}}$, as a function of $t_{\mathrm{snr}}$. As in the top panel, the solid curves are from our CR-hydro model and the dashed curve (FS only) is from Berezhko et al. (2002). The total $E_{\mathrm{cr}} / E_{\mathrm{sn}}$ is in good agreement at early times but diverges somewhat at later times, a possible indication that adiabatic losses are treated differently in the two models. Most importantly, both models show that approximately $50 \%$ of the explosion energy is placed into cosmic rays during the SNR lifetime ${ }^{13}$. The dotted curves in the bottom panel show that $E_{\mathrm{cr}} / E_{\mathrm{sn}}$ at $t_{\mathrm{snr}}=6000 \mathrm{yr}$ remains in a fairly narrow range ( $\sim 40$ to $65 \%$ ) for $\eta_{\text {inj }}$ ranging from $8 \times 10^{-5}$ (a), to $10^{-3}(\mathrm{~d})$.

\section{Conclusions}

The efficient production of cosmic rays by shocks in SNRs lowers the pressure to energy density ratio in the post-shock gas causing dramatic changes in the thermal properties of the shocked gas and the SNR evolution. We have presented a CR-hydro model that combines a 1D hydrodynamic simulation of a SNR, including the forward and reverse shocks, with particle acceleration. We explicitly include the effects of particle acceleration on the shock heated ejecta, a critical step in determining how X-ray thermal emission from the hot ejecta is modified by particle acceleration.

In accord with previous results (e.g., Dorfi 1990; Berezhko et al. 1996, 2002), we find that SNRs can easily transfer $\sim 50 \%$ of the explosion energy into relativistic particles (bottom panel of Fig. 11). Compared to the situation where acceleration is

\footnotetext{
13 Berezhko et al. (2002) make the important point that injection may vary over the surface of the SNR and be significantly less where the magnetic field is highly oblique. They estimate that to supply the galactic cosmic-ray population the overall efficiency need only be $\sim 20 \%$ of the maximum values shown in Fig. 11 .
}

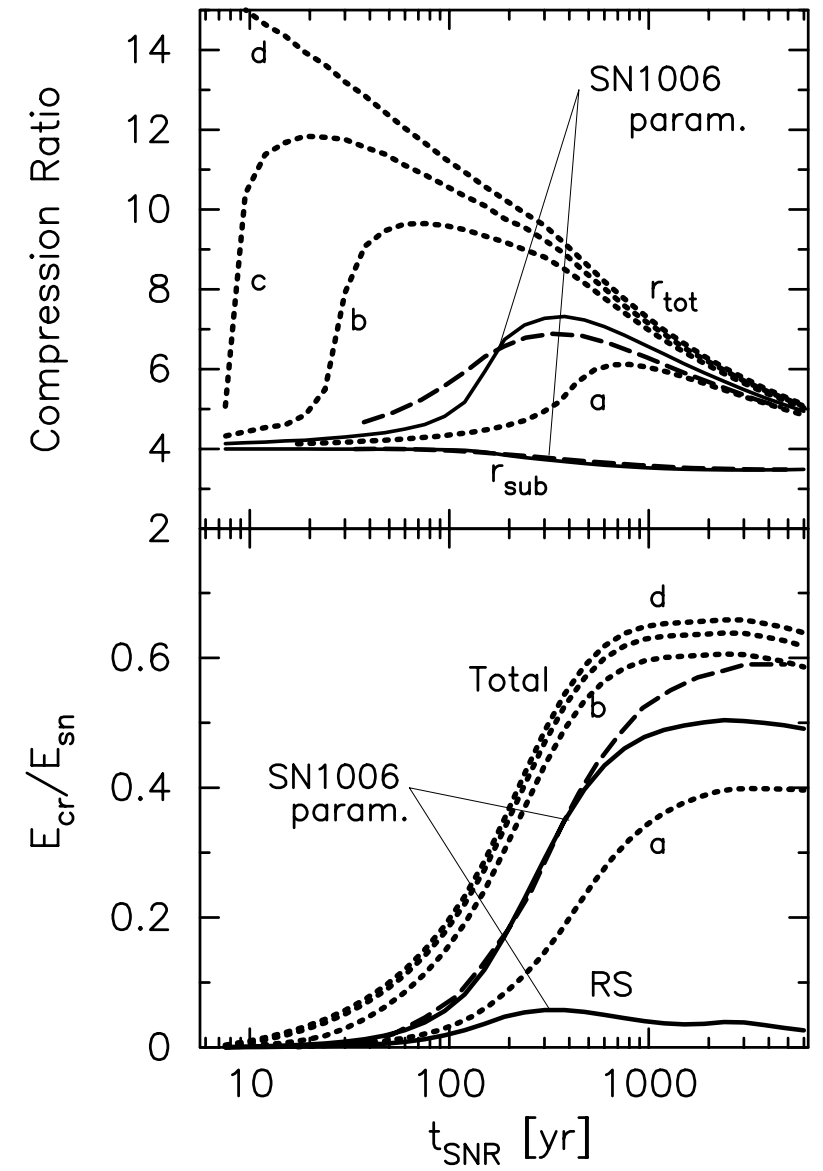

Fig. 11. Forward shock compression ratios (top panel) and fraction of $E_{\mathrm{sn}}$ going into relativistic particles, $E_{\mathrm{cr}} / E_{\mathrm{sn}}$, (bottom panel) for SNRs with various $\eta_{\text {inj }}$ 's versus $t_{\text {snr. }}$. The solid curves are for SN1006 parameters $\left(\eta_{\mathrm{inj}}=1.2 \times 10^{-4}, B_{0}=20 \mu \mathrm{G}, E_{\mathrm{sn}}=3 \times 10^{51} \mathrm{erg}\right.$, $M_{\mathrm{ej}}=1.4 M_{\odot}, n_{\mathrm{p} 0}=0.3 \mathrm{~cm}^{-3}$, and $\left.f_{\mathrm{He}}=0.1\right)$ and show $r_{\text {tot }}$ and $r_{\mathrm{sub}}$ in the top panel and the total $E_{\mathrm{cr}} / E_{\mathrm{sn}}$ and that portion from the RS in the bottom panel. The dashed curves are the corresponding SN1006 results from Berezhko et al. (2002). The dotted curves are from the CR-hydro model with a) $\eta_{\text {inj }}=8 \times 10^{-5}$, b) $\eta_{\text {inj }}=2.4 \times 10^{-4}$, c) $\eta_{\text {inj }}=4 \times 10^{-4}$, and d) $\eta_{\text {inj }}=10^{-3}$.

absent or inefficient, this transfer results in lower FS speeds, larger overall compression ratios, cooler post-shock temperatures, and a smaller and denser interaction region between the forward and reverse shocks. At young ages, compression ratios $r_{\text {tot }}>6$ (top panel of Fig. 11) and proton temperatures less than $\lesssim 1 / 10$ the TP value are predicted (Fig. 6) for the particular set of SN and ISM parameters used here to model SN 1006. For some injection efficiencies, an abrupt transition is predicted to occur at early times $\left(t_{\mathrm{snr}}<t_{\mathrm{ch}}\right)$ between high Mach number, unmodified shocks with $r_{\text {tot }} \sim 4$ and strongly modified shocks with $r_{\text {tot }} \gg 4$ (top panel of Fig. 11), causing steep spatial gradients in temperature (Fig. 4). We believe the changes in shock speed, density, temperature, and spatial extent and profile are large enough to (i) provide diagnostics for X-ray observations of young SNRs sufficient to place meaningful constraints on the acceleration efficiency, and (ii) to importantly modify the inferred values of $E_{\mathrm{sn}}, M_{\mathrm{ej}}$, and ambient ISM density once a full 
thermal X-ray emission model is combined with the CR-hydro model.

Despite the strong NL effects expected for young SNRs, the CR spectrum integrated over the age of a remnant (Fig. 9) should have a spectrum not much flatter than $N(E) \propto E^{-2}$ at relativistic energies.

Our one-dimensional CR-hydro model uses a computationally fast, approximate calculation of particle acceleration which is coupled to the hydrodynamics by modifying the effective ratio of specific heats. We have verified the accuracy of this approach for parameters applicable to SN1006 by direct comparison with the more physically complete model of acceleration of Berezhko et al. (2002) (Figs. 10 and 11), where the time-dependent CR transport equations are solved selfconsistently with the gas-dynamic equations. The main advantage of our model lies in the fact that we can model acceleration at the forward and reverse shocks during all stages of the SNR evolution. In a preliminary work we modeled thermal Xray emission from Kepler's SNR with a two-fluid, self-similar description of the SNR hydrodynamics coupled to the same calculation of particle acceleration (Decourchelle et al. 2000). We have demonstrated (Fig. 7) that the two models give essentially identical results when self-similar conditions apply. Our next step will be to include this non-equilibrium calculation of thermal X-ray emission, plus broad-band continuum emission from bremsstrahlung, synchrotron, inverse-Compton, and pion-decay (Baring et al. 1999), in the CR-hydro model.

Acknowledgements. The authors would like to thank J. Blondin for providing his hydrodynamic simulation $\mathrm{VH}-\mathrm{l}$ and for help modifying it to include particle acceleration. This work was supported, in part, by a NSF-CNRS grant (NSF INT-0128883) and by a NASA ATP grant (ATP02-0042-0006).

\section{References}

Allen, G. E., Keohane, J. W., Gotthelf, E. V., et al. 1997, ApJ, 487, L97

Baring, M. G., Ellison, D. C., Reynolds, S. P., Grenier, I. A., \& Goret, P. 1999, ApJ, 513, 311

Berezhko, E. G., \& Ellison, D. C. 1999, ApJ, 526, 385

Berezhko, E. G., Elshin, V. K., \& Ksenofontov, L. T. 1996, JETP, 82,1

Berezhko, E. G., \& Krymsky, G. F. 1988, Sov. Phys. Usp., 31, 27

Berezhko, E. G., Ksenofontov, L. T., \& Petukhov, S. I. 1999, Proc. 26th Int. Cosmic Ray Conf. (Salt Lake City), 99, 4431

Berezhko, E. G., Ksenofontov, L. T., \& Völk, H. J. 2002, A\&A, 395, 943

Berezhko, E. G., Pühlhofer, G., \& Völk, H. J. 2003, A\&A, 400, 971

Berezhko, E. G., \& Völk, H. J. 1997, Astropart. Phys., 7, 183

Blandford, R. D., \& Eichler, D. 1987, Phys. Rep., 154, 1
Blasi, P. 2002, Astropart. Phys., 16, 429

Blondin, J. M., \& Ellison, D. C. 2001, ApJ, 560, 244

Borkowski, K. J., Rho, J., Reynolds, S. P., \& Dyer, K. K. 2001, ApJ, 550,334

Boulares, A., \& Cox, D. P. 1988, ApJ, 333, 198

Butt, Y. M., Torres, D. F., Romero, G. E., et al. 2002, Nature, 418, 499

Chevalier, R. A. 1982, ApJ, 258, 790

Chevalier, R. A. 1983, ApJ, 272, 765

Decourchelle, A., \& Ballet, J. 1994, A\&A, 287, 206

Decourchelle, A., \& Ellison, D. C. 2001, Space Sci. Rev., 99, 219

Decourchelle, A., Ellison, D. C., \& Ballet, J. 2000, ApJ, 543, L57

Dorfi, E. A. 1990, A\&A, 234, 419

Dorfi, E. A. 1994, ApJS, 90, 841

Dorfi, E. A., \& Böhringer, H. 1993, A\&A, 273, 251

Drury, L. O'C. 1983, Rep. Prog. Phys., 46, 973

Drury, L.O'C., Ellison, D. E., Aharonian, F. A., et al. 2001, Space Sci. Rev., 99, 1-4, 329

Ellison, D. C., Berezhko, E. G., \& Baring, M. G. 2000, ApJ, 540, 292

Ellison, D. C., \& Eichler, D. 1984, ApJ, 286, 691

Ellison, D. C., Slane, P., \& Gaensler, B. M. 2001, ApJ, 563, 191

Enomoto, R., Tanimori, T., Naito, T., et al. 2002, Nature, 416, 823

Ghavamian, P. 1999, Ph.D. Thesis, Rice University

Giacalone, J., Burgess, D., Schwartz, S. J., \& Ellison, D. C. 1993 , ApJ, 402, 550

Heavens, A. F. 1984, MNRAS, 211, 195

Hughes, J. P., Rakowski, C. E., \& Decourchelle, A. 2000, ApJ, 543, L61

Hwang, U., Decourchelle, A., Holt, S. S., \& Petre, R. 2002, ApJ, 581, 1101

Jokipii, J. R., Kóta, J., \& Giacalone, J. 1993, G.R.L., 20, 1759

Jones, F. C., \& Ellison, D. C. 1987, JGR, 92, 11,205

Jones, F. C., \& Ellison, D. C. 1991, Space Sci. Rev., 58, 259

Jones, F. C., Jokipii, J. R., \& Baring, M. G. 1998, ApJ, 509, 238

Kazanas, D., \& Ellison, D. C. 1986, ApJ, 304, 178

Koyama, K., Petre, R., Gotthelf, E. V., et al. 1995, Nature, 378, 255

Laming, J. M., Raymond, J. C., McLaughlin, B. M., \& Blair, W. P. 1996, ApJ, 472, 267

Long, K. S., Reynolds, S. P., Raymond, J. C., et al. 2003, ApJ, 586, 1162

Malkov, M. 1997, ApJ, 485, 638

Malkov, M. 1998, Phys. Rev. E, 58, 4911

Malkov, M. A., Diamond, P. H., \& Jones, T. W. 2002, ApJ, 571, 856

Malkov, M. A., \& Drury, L. O'C. 2001, Rep. Prog. Phys., 64, 429

Raymond, J. C., Blair, W. P., \& Long, K. S. 1995, ApJ, 454, L31

Reimer, O., \& Pohl, M. 2002, A\&A, 390, L43

Reynolds, S. P. 1998, ApJ, 493, 375

Reynolds, S. P., \& Ellison, D. C. 1992, ApJ, 399, L75

Slane, P., Gaensler, B. M., Dame, T. M., et al. 1999, ApJ, 525, 357

Truelove, J. K., \& McKee, C. F. 1999, ApJS, 120, 299

Völk, H. J., Berezhko, E. G., Ksenofontov, L. T., \& Rowell, G. P. 2002, A\&A, 396, 649 